\title{
Philippe Sagant, la passion de l'ethnologie
}

Katia Buffetrille et Marie Lecomte-Tilouine

\section{(2) OpenEdition}

\section{Journals}

Édition électronique

URL : https://journals.openedition.org/emscat/2721

DOI : 10.4000/emscat.2721

ISSN : 2101-0013

Éditeur

Centre d'Etudes Mongoles \& Sibériennes / École Pratique des Hautes Études

\section{Référence électronique}

Katia Buffetrille et Marie Lecomte-Tilouine, «Philippe Sagant, la passion de l'ethnologie », Études mongoles et sibériennes, centrasiatiques et tibétaines [En ligne], 46 | 2015, mis en ligne le 10 septembre 2015, consulté le 13 juillet 2021. URL : http://journals.openedition.org/emscat/2721 ; DOI : https:// doi.org/10.4000/emscat.2721

Ce document a été généré automatiquement le 13 juillet 2021.

(c) Tous droits réservés 


\title{
Philippe Sagant, la passion de l'ethnologie
}

\author{
Katia Buffetrille et Marie Lecomte-Tilouine
}

\section{NOTE DE L'AUTEUR}

Hommage dirigé par Katia Buffetrille et Marie Lecomte-Tilouine

Photographies de Katia Buffetrille

Philippe Sagant est né le 8 mai 1936 et nous a quittés le 10 janvier 2015, après une très longue et cruelle maladie. Nous avons voulu ici retracer les jalons de sa carrière et de sa vie intellectuelle, avant de laisser la parole à quelques uns de ses collègues et étudiants.

2 Après des études à l'EPHE, Philippe Sagant soutient en 1973 une thèse de doctorat intitulée "Le paysan limbu, sa maison et ses champs ", à l'université de Paris VII, sous la direction de Lucien Bernot (EHESS). Affecté au Museum d'Histoire Naturelle/Musée de l'Homme en 1965, il intègre le CNRS en 1966 comme attaché de recherche en ethnologie, puis promu chargé de recherches en 1974, il termine sa carrière comme directeur de recherches au CNRS.

3 Membre du Centre d'études de l'Inde et de l'Asie du Sud à partir de 1970, Philippe Sagant a dispensé des enseignements de 1978 à 1983 à l'Université de Haute-Bretagne, Rennes, ainsi qu'à l'Université Paris $\mathrm{X}$, Nanterre, au sein du séminaire de maîtrise d'A. Macdonald, « Le domaine himalayen ", et à l'INALCO, où il fut chargé de cours sur la civilisation népalaise de 1981 à 1993.

4 Ses enquêtes de terrain ont tout d'abord mené Philippe Sagant en Aubrac, puis il se spécialisa sur le Népal où il fit de nombreux séjours entre 1966 et 1986. Il choisit d'étudier un petit groupe ethnique de langue tibéto-birmane vivant à la frontière orientale du pays: les Limbu. La majeure partie de son œuvre s'appuie sur les matériaux réunis en "Pays Limbu", avec, tout d'abord, sa thèse de doctorat, qui a formé la matière de son ouvrage de référence: Le paysan limbu, sa maison et ses champs 
(Mouton 1976). Nous laisserons ici A. Macdonald, son collègue et ami, en souligner les qualités exceptionnelles :

[O]n a ici le tableau le plus exact et le plus vivant de la vie des paysans des collines de l'est du Népal. [...] P. Sagant [...] constate « l'apparition d'une société à tendance de classe qui s'articule sur les clivages de castes et d'ethnies » (p. 273) et soumise à l'autorité administrative de Kathmandou. Il explique ce bouleversement social par la transformation profonde du milieu technique : l'outillage, dominé par l'araire, est actuellement identique pour toutes les populations des collines et on pratique partout la même agriculture. [...] [I]l nous fait comprendre de façon passionnante « la lutte pour la terre » et l'enjeu de la compétition foncière [...]. Sur la maison et l'agriculture limbu, P. Sagant nous a donné un ouvrage définitif. J'espère qu'il ne m'en voudra pas de suggérer qu'il nous a aussi donné un livre essentiel pour la compréhension de l'hindouisation politique de l'est du Népal. ${ }^{1}$

5 Parti de l'étude de l'agriculture, de l'outillage et du foncier, Philippe Sagant va rapidement se révéler un fin anthropologue du religieux et du politique. Son intime connaissance du contexte villageois le conduira à se démarquer radicalement des études jusque là menées sur le chamanisme, en montrant qu'une séance ne doit pas se comprendre comme un tout, mais doit être replacée dans un parcours de vie où prime l'interprétation collective des événements. Dans cette région où les chefs règnent littéralement sur de petits territoires comme des roitelets, il aura à cœur de comprendre le fonctionnement de ce morcellement, et sa formation, ainsi que le rôle de l'assemblée des chefs, et enfin s'interrogera sur la nature de l'autorité du chef en ce milieu composite, entre chamanisme animiste et hindouisme. Il soulignera le rôle de la chasse, celui de la force vitale, des dieux des montagnes, ainsi que des formes locales de la déesse. Ces thèmes le conduiront tout naturellement à se tourner vers le Tibet, et il choisit de les soumettre à un informateur de choix: Samten Karmay. Tous deux décidèrent alors d'écrire un ouvrage ensemble. En 1986-87, Philippe souhaita compléter son enquête par l'observation ethnographique, et se rendit dans le village de Samten, au Tibet, en compagnie de Katia Buffetrille. Il voulait rencontrer les hommes, voir le village et les temples, s'imprégner du paysage et de l'atmosphère de ces lieux qu'il ne connaissait alors qu'à travers les paroles de ce dernier. Travaillant en étroite collaboration sur le thème de l'autorité traditionnelle au Tibet, ils ont montré de manière convaincante que «dans certaines régions, le chef dérive son autorité et sa légitimité des dieux du territoire » (Ramble 2006, p. 6). ${ }^{2}$

Suite à ce séjour au Tibet, Philippe Sagant poursuivit son enquête au Népal, dans le district de Manang, qui devait être son dernier terrain ethnographique, et qui a été la source d'un bel article où il montre les relations entre culte aux montagnes sacrées et organisation politique.

7 Enfin, certains des travaux de Philippe Sagant ont été rendus accessibles à un plus grand nombre de lecteurs par la parution, en 1996, aux éditions Oxford University Press, d'une collection de ses articles traduits en anglais, sous le titre de l'un de ses meilleurs essais: The Dozing Shaman. On ne peut qu'espérer que l'ensemble de son œuvre, qui figure ci-dessous en bibliographie, soit ainsi diffusé en anglais très prochainement. 


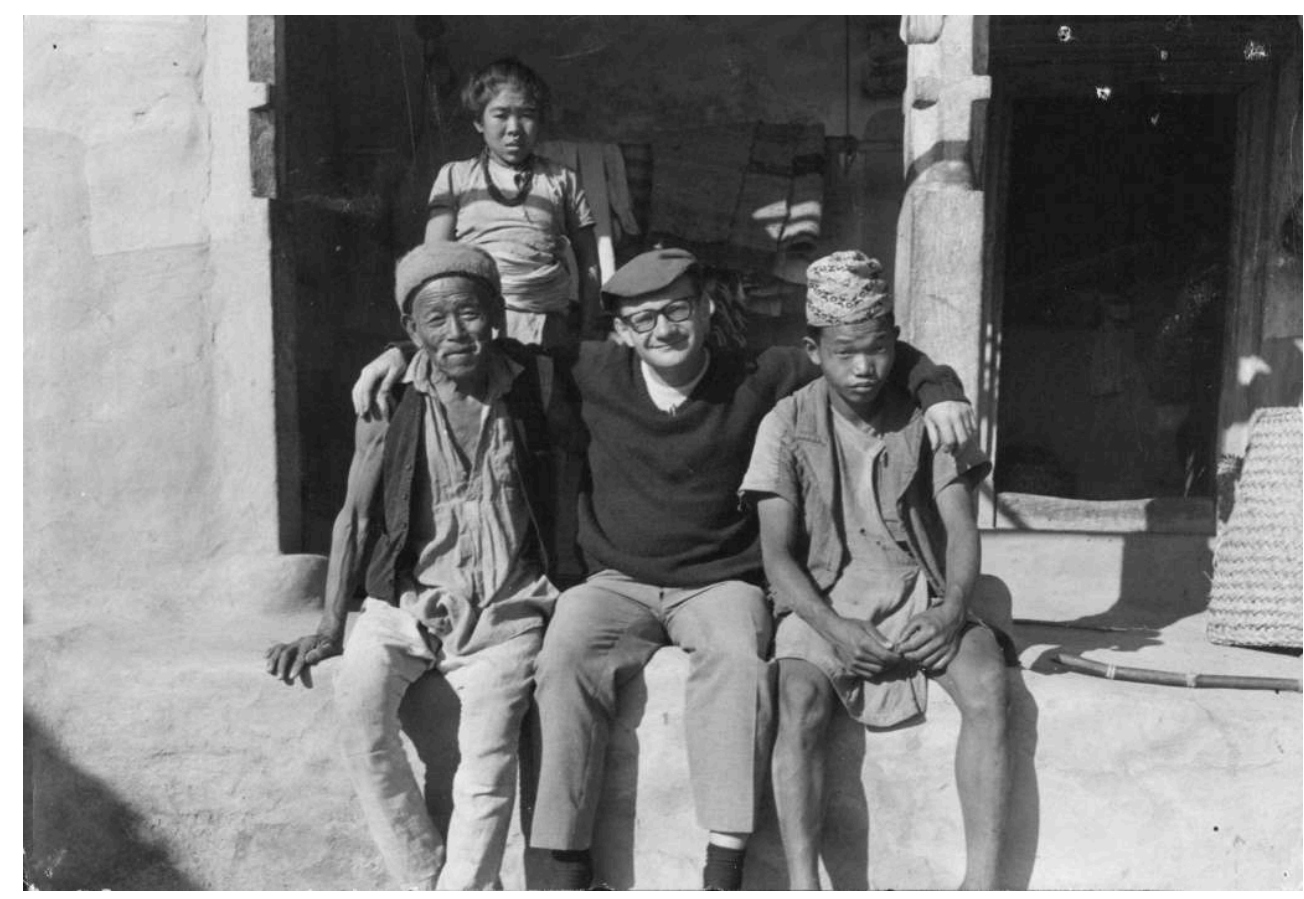

Philippe Sagant en compagnie d'amis Limbu à Libang (date inconnue)

Le système thekka thiti avait pour but d'isoler les chefs limbu les uns des autres, de casser leurs relations politiques, de les placer sous le contrôle direct et exclusif de la cour régionale (adâlat) et du bureau des revenus fonciers (mal adda). On les vit au contraire, par leurs fonctions de juges, constituer sur l'ensemble du pays un réseau serré de relations personnelles et hiérarchisées : une féodalité.

Sans doute auraient-ils pu tenir longtemps. Toutefois, les mesures prévoyant l'intégration des immigrés sur les terres du segment de clan ouvrirent le chemin à la pénétration des Népalais au plus profond du pays limbu. Précipitant les transformations du milieu technique et économique, ce sont elles, en fin de compte, qui permirent à l'État gurkha d'en finir avec la résistance limbu. ${ }^{3}$

8 Pour Philippe Sagant, le rôle de l'ethnologue consistait à montrer comment une société se comprenait elle-même, puis à le transmettre aux autres. Il aimait établir des relations personnelles aussi bien sur le terrain que dans la vie quotidienne et nombreux sont ceux qui ont été profondément marqués par son enseignement, par son amitié. Certains d'entre eux ont tenu à l'exprimer. 


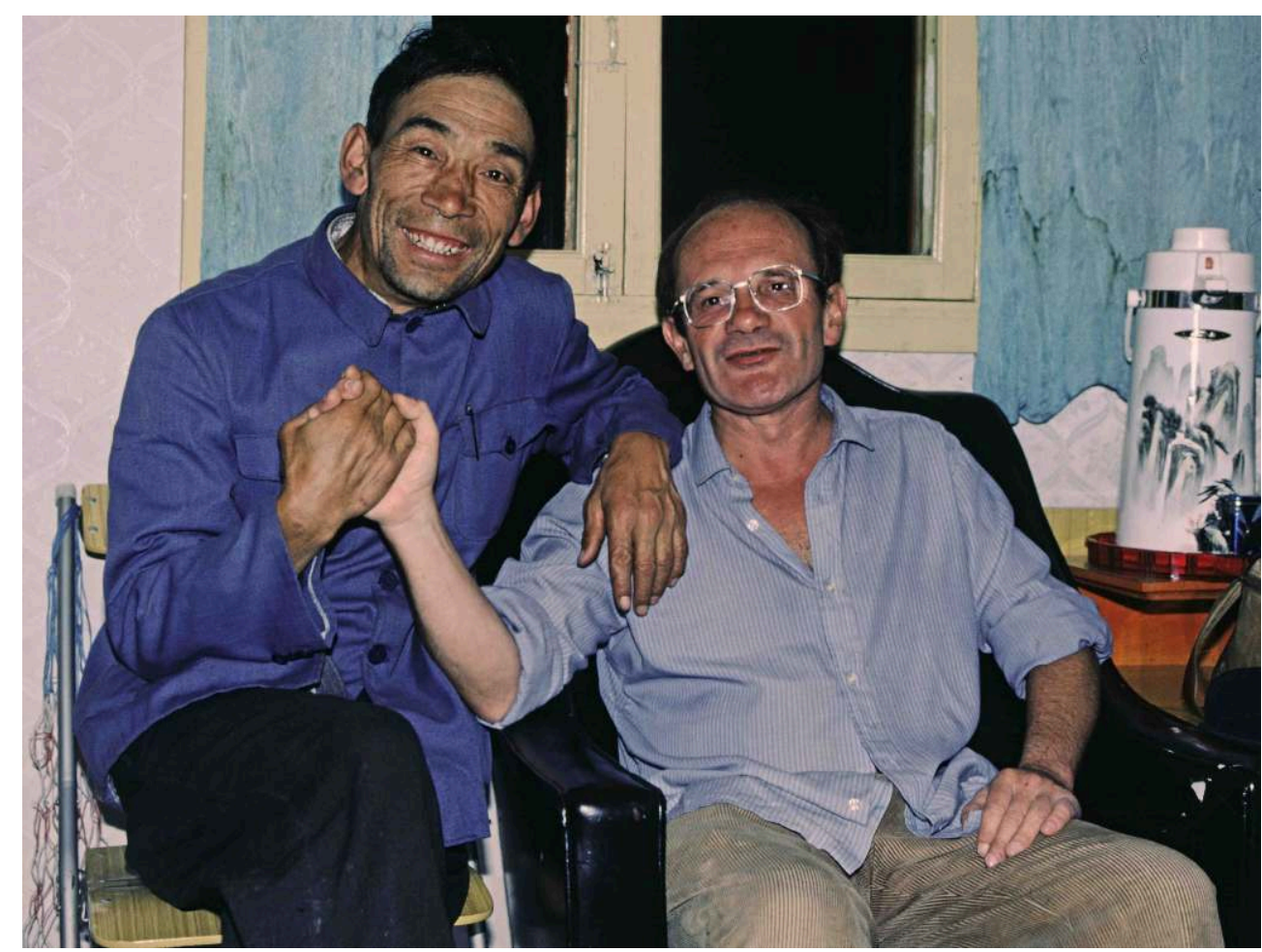

Katia Buffetrille (1986)

Sur le terrain, tout le travail de l'ethnologue consiste à donner du sens à ce qu'il voit, non pas le sens que lui, l'Occidental, croit pouvoir discerner, mais celui qu'attribuent les indigènes eux-mêmes. Alors seulement, peut-être, apparaissent les enjeux. ${ }^{4}$

\section{András Höfer}

My acquaintance with Philippe Sagant dates back to the year 1964 when we were both attending a class of the unforgettable Lucien Bernot, held in the sous-sol of a building in rue Monsieur le Prince in Paris. Mutual sympathy and a common dilemma soon brought us closer together. We had both started specialising in the ethnology of Mainland Southeast Asia and were at the same time fully aware of the impossibility of carrying out fieldwork among any of the ethnic minorities in the then-restricted areas of Assam, Arunachal Pradesh or Burma.

One day, while we were having a drink in one of the cafés of boulevard Saint Michel, Philippe revealed to me, out of the blue, his decision to relinquish his aspirations regarding Southeast Asia and try research in Nepal instead. He also suggested that I should join him in a course of colloquial Nepali, that was taking place on the top floor of the Musée de l'Homme and run by a maitre de recherche by the name of Macdonald. That's how I came to embark on Nepal studies.

11 A few years later, I bumped into Philippe in Kathmandu. I had just come back from the hills, and our long, cheerful conversation inevitably pivoted around our practical experiences " out there " in our respective fields. At a certain point, I somehow felt compelled to give our talk a - perhaps rather unbecoming - academic twist by broaching the question of what exactly his current fieldwork in Limbu country was aiming at. I still recall, more or less literally, the wording of the reply Philippe gave me. He said, «Well, you know, I frequently withdraw into a nearby forest with a couple of villagers in my company ; there we sit down, drink plenty of millet spirit, and I listen to 
the stories of the people. I'm interested in those micro-détails [a favourite term of Lucien Bernot] contained in their narrations. » I resisted the temptation to demand more. Inasmuch as Philippe's modesty, tinted with subtle self-irony, was notorious, I had no reason not to suspect something promising behind his understatement. And yet, who would have thought at that time that all these détails were already maturing like grains of sand to pearls and would a little later gather momentum to reappear in surprisingly telling contexts in his studies, such as, say, «Le chamane assoupi » or «Le paysan limbu, sa maison et ses champs ", all so well-founded and unique in their approach ?

\section{Michael Oppitz, The dozing ethnographer}

If I remember correctly, I had met Philippe through Rolf Stein and Sandy Macdonald. Stein had given a course at the Collège de France on the phurbu, which we both attended. When we strolled along in the streets of Paris - must have been quite a number of times - we talked about our constant brooding concerning form : how to write ethnography. Philippe had found it for his book on the Limbu peasant. For me his descriptions of village life as determined by the seasonal changes from month to month were just perfect. Another topic of our talk was loneliness in the field. We both confessed that we had been reading trashy novels (Malinowski) to combat it - I had devoured Joyce's Ulysses on the porch of a Nyingmapa monastery in Rolwaling; Philippe had been distracted by Flaubert up in Libang. And we both confessed that rituals could at times be so tiresome that not only the village audience and some of the main protagonists would fall asleep, but also the most avid ethnographer. Was it this experience that gave rise to Philippe's most famous title?

\section{Charles Ramble}

The academic milieu can seem like a mildly threatening environment at the best of times, but especially so for anyone who has been out of touch with it for a while. When, after several years of absence, I began to make faltering steps at reintegration, it was in France in the early 1990s. Among the people who convinced me that the effort would be worthwhile was Philippe Sagant. At this time he was becoming increasingly interested in trans- and cis-Himalayan comparisons, but although he had clearly read very widely and had developed numerous ideas on the subject, he made no attempt to set me on any theoretical or methodological tramlines. Over the course of coffees and lunches he just got me to talk about what I had seen and done in Tibet and the Himalaya. Talking about ethnography is not the same thing as writing it ; but it does mean that you have to think about it, and, in thinking about it, subject it to a first round of organisation. There is nothing more encouraging that talking to someone who is interested in what you have to say. It makes everything more real. Spending time with Sagant made me realise that listening to stories is not just a passive exercise but, when intelligently done, has a reciprocal effect on both the story and the narrator. Philippe Sagant was an original thinker and a fine writer, but he was also an exceptionally creative listener.

\section{Marc Gaborieau}

Mon amitié avec Philippe Sagant remonte à 1965. Cette année-là Jacques Millot, directeur du Musée de l'Homme, et Corneille Jest, chargé de recherche au CNRS, mirent sur pied une équipe de recherche sur le Népal. Philippe Sagant et moi-même avons été parmi les premiers jeunes chercheurs intégrés dans cette équipe qui permit notre recrutement au CNRS. Philippe vint alors faire sa première mission de longue durée chez les Limbu du Népal oriental : il séjourna longuement chez moi à Katmandou à l'automne 1966 pour préparer son départ sur le terrain et à l'été 1967 à son retour. 
Durant ces semaines de séjour forcé à Katmandou, notre amitié s'est forgée et nos discussions se sont réellement engagées sur nos conceptions de l'ethnologie au départ divergentes mais destinées à converger. Nous avons eu ensuite l'opportunité de collaborer plus longuement à l'EHESS, dans le cadre de l'équipe «Village » du Centre d'Etudes de l'Inde et de l'Asie du Sud de 1974 à 1978, qui donna lieu à deux publications collectives. Dans la première (L'Homme XVIII ${ }^{1-2}$, 1978, pp. 109-134), Philippe Sagant donna une magistrale analyse des "pouvoirs de chefs limbu au Népal oriental». Il assuma ensuite la responsabilité éditoriale d'un numéro spécial de L'ethnographie (CXX année, 1978, II, Nlle série, ${ }^{\circ}$ 77-78) sur « Les migrations dans l'Asie du Sud ». Il donna enfin une intrigante communication sur « Le chamane assoupi » au colloque Asie $d u S u d^{5}$. Nos chemins devaient ensuite se séparer, mais je garde le souvenir d'un ethnologue original qui a marqué l'orientation de mes recherches et qui est resté un ami fidèle sur lequel on pouvait compter.

\section{charles Malamoud}

Je suis un lecteur de textes : textes sanscrits, pour la plupart, composés il y a vingt-cinq siècles par des brahmanes, pour des brahmanes. Il se trouve cependant que dès mes premiers pas dans les études indiennes, j'ai croisé ou côtoyé des hommes et des femmes qui voyaient l'Inde sous un tout autre angle: ethnologues, ils étudiaient des communautés bien circonscrites, bien déterminées par leur environnement immédiat, vivantes, et dont, pour de longues périodes de "terrain ", ils partageaient la vie. Il se trouve aussi que la plupart de ces chercheurs, surcroît d'exotisme pour moi, étudiaient non pas l'Inde proprement dite mais sa bordure himalayenne. Parmi eux, Philippe Sagant.

16 J'ai participé avec lui à diverses rencontres et réunions, surtout des jurys, au Laboratoire d'Ethnologie de l'Université de Paris X Nanterre, dans les années 1970. Les circonstances n'étaient pas propices à des conversations en tête-à-tête, mais ces discussions m'ont permis de découvrir en Philippe Sagant, doublant le chercheur rigoureux et original, un homme vraiment humain, constamment soucieux des moyens d'existence et des conditions de travail des étudiants que nous devions certes orienter et évaluer, mais aussi encourager et soutenir. Mais surtout le souvenir que je garde de Philippe Sagant est tout entier habité par la gratitude: dans le numéro de la revue Purușārtha que j'ai dirigé et qui est consacré à « la dette » dans le monde indien $\left(\mathrm{n}^{\circ} 4\right.$, 1980), figure un article que Philippe Sagant a bien voulu nous donner. A la fois ample, précis et profond, il comporte une typologie de la dette qui découle d'une analyse des modes d'endettement en pays Limbu, liée elle-même à l'histoire de l'hindouisation de ces montagnes, et donc de l'introduction du système des castes. C'était une façon de " situer » et de mettre en perspective, dans le temps et dans l'espace, l'idée de " dette " que je m'étais efforcé de mettre en lumière dans les textes surtout normatifs de l'Inde brahmanique. Au moment où il nous quitte, j'ai à cœur de rappeler ce qui demeure ma propre dette à l'égard de Philippe Sagant.

17 Boyd Michailovsky

En 1976 j'ai demandé conseil à Philippe Sagant pour une enquête sur la langue limbu, et il m’a très généreusement donné le nom du village, Libang, où il avait le plus travaillé : "Je te donne un seul nom, 'Motta' - Harka Jit. Allez le voir!" Nous avons donc passé deux mois chez lui en pleine mousson et recueilli les récits en langue limbu qui sont consultables sur le site Pangloss actuellement. L'un de ces textes a inspiré à Philippe un article merveilleux sur la malemort qu'il m'a fait co-signer en 1992. 
Ce dont je me rappelle de Philippe est sa gentillesse, et le plaisir qu'on avait à recevoir les petits mots amicaux, manuscrits, qu'il envoyait par la poste.

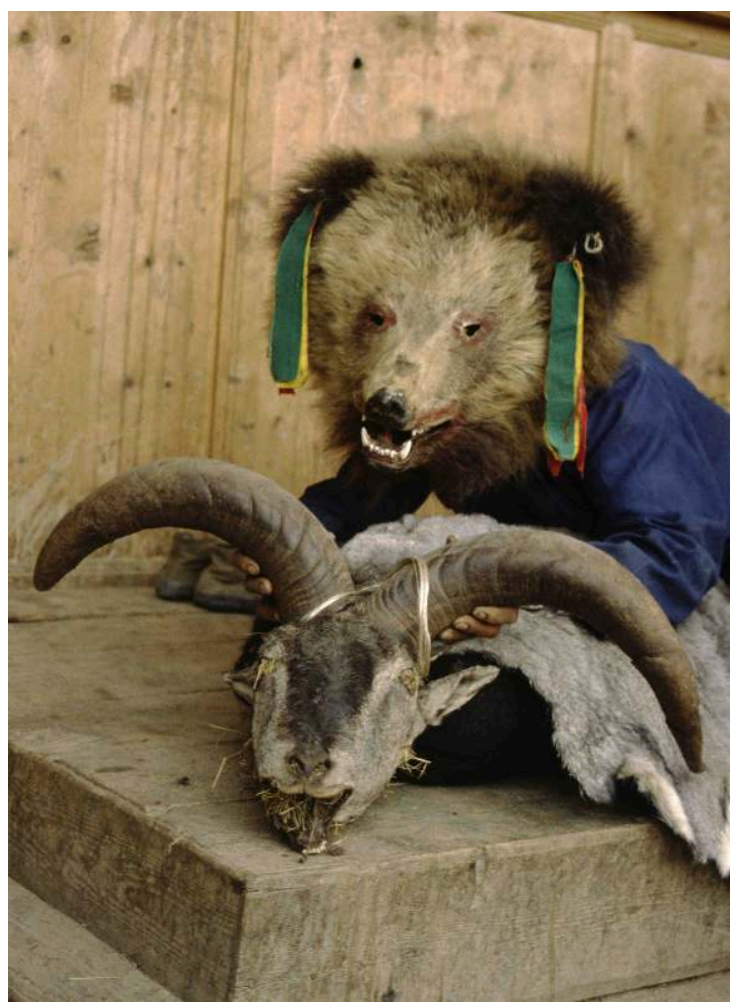

Katia Buffetrille (1986)

Parce que j'étais curieux des gibiers qu'il chassait, Tendar nous entraîna sur la terrasse de sa maison. Sous les toits étaient amoncelés des trophées de toutes sortes. Du haut d'une échelle, Dargyé les sortait un à un, ça n'en finissait plus: têtes de moutons sauvages avec cornes gigantesques, massacres de cerfs aux vastes andouillers, énormes crânes d'ours empaillés, dépouilles d'ovins et de caprins sauvages dont je serais en peine de dire les noms...

\section{Pascal Bouchery}

Philippe Sagant a profondément marqué la génération d'himalayanistes à laquelle j'appartiens. Tout d'abord par le style de ses écrits, remarquable entre tous. Il parvenait à dépeindre la complexité des réalités sociales dans un style incisif d'une très grande sobriété, et déroulait son propos comme on raconte une histoire. C'est ensuite par la puissance de ses idées que Philippe Sagant forçait l'admiration, résultat d'une subtile alchimie entre Granet, Hocart et Marx. L'Asie lui apparaissait comme un tout, il traquait dans des sociétés très diverses la présence de thèmes récurrents (la force vitale, l'élection divine, le double pouvoir, la place de rang, les chasses rituelles, le jeu...) qui lui paraissaient de nature à s'ordonner en système et, ce faisant, étaient propices à révéler des logiques sociales similaires. Enfin Philippe Sagant était un ethnographe hors pair, particulièrement méticuleux dans la réalisation de croquis et dessins. Sa pensée et ses intuitions souvent géniales continueront encore longtemps de nous inspirer.

\section{Anne de Sales}

Philippe Sagant aimait les histoires, les écouter et les raconter. Il rappelle dans son introduction à l'édition anglaise de ses essais, The Dozing Shaman, que c'est en écoutant 
les récits quotidiens par lesquels les Limbus relataient les événements de leur vie, qu'il avait l'impression d'atteindre au plus près l'image qu'ils se faisaient d'eux-mêmes, de leurs institutions et du monde autour d'eux. Il était très attentif à la qualité narrative de l'expérience des individus. Ainsi avant mon départ pour mon premier terrain, chez les Kham-Magar, il m'a donné un conseil dont je lui serais toujours redevable : « quand tu arriveras au village, dis-toi que tu dois rester un mois, c'est tout. Au bout de ce premier mois, tu pourras reconsidérer l'affaire ». Sans ce conseil je me serais aussitôt enfuie, ne supportant pas la solitude à laquelle les villageois d'abord méfiants et peu hospitaliers m'avaient réduite. Mais un mois, j'ai pensé que je pourrais tenir. Et je suis restée dix-huit mois. En me racontant le début de mon histoire avant que je la vive, Philippe m'avait mis sur le chemin.

\section{Philippe Ramirez}

Quelque fin d'après-midi autour de 1985-86, un café à Montparnasse, deux demis, deux Philippe face à face, séance de debriefing: un Philippe terrorisé, attendant le verdict quant à deux pages de bafouilles, sur la vendetta; l'autre Philippe, tranquillement, lui tendant huit pages de commentaires, et démarrant, aussi timide sûrement, mais avec ce petit sourire narquois et bonhomme, et tout de suite la passion, la passion pour le cru, pour le vu, pour les connexions, pour les comparaisons, pour «l'ethno " - comme il disait avec respect. Au café, à Nanterre, aux Langues'O, tu revenais obstinément sur le trésor que tu avais découvert là-bas: "La honte ça peut faire mourir, il faut faire sa place ». Tu en as fait une énorme, et tu l'as fait tranquillement. Salut mon Prof, salut Philippe.

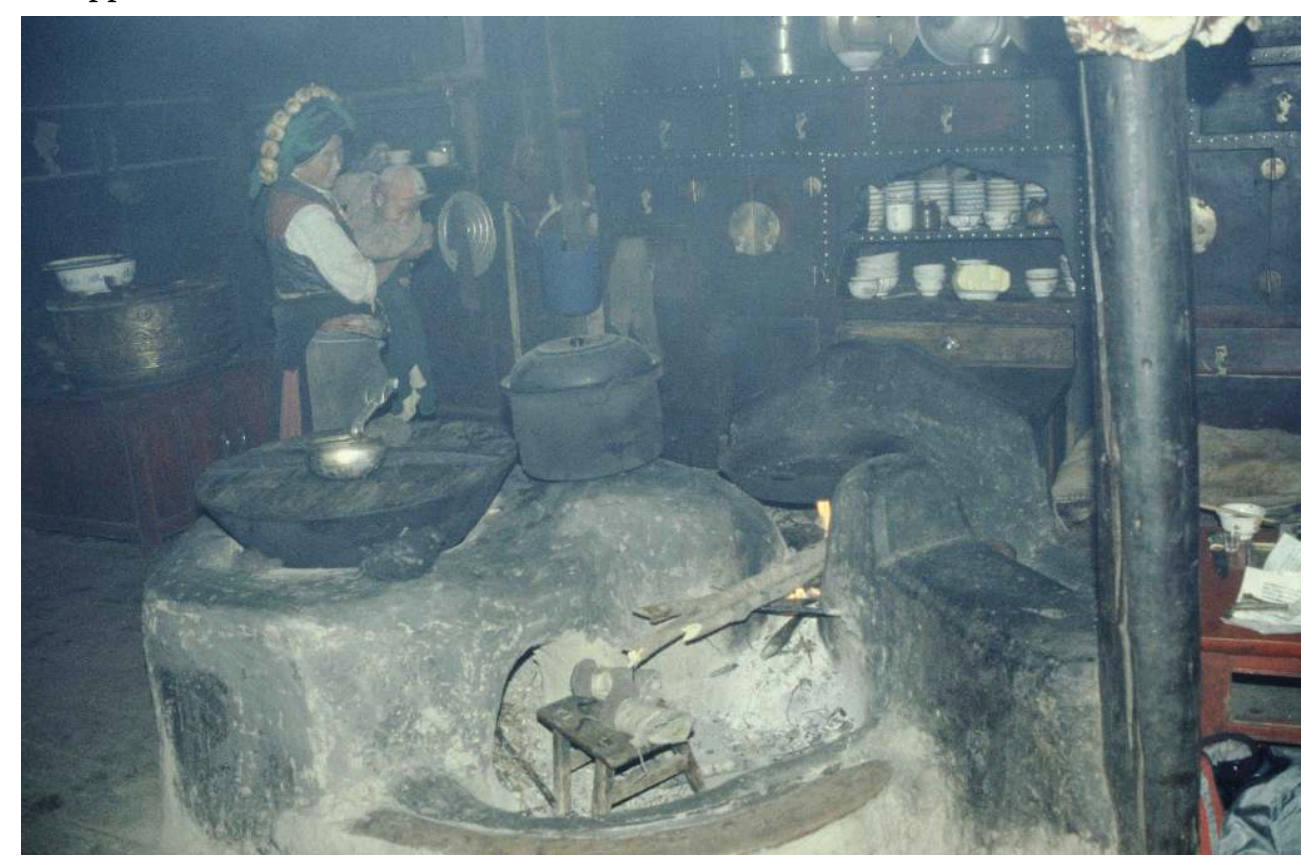

Katia Buffetrille (1986)

Le foyer (thab ka) est une construction monumentale qui prend appui au fond sur le mur ouest et déborde largement jusqu'au milieu de la pièce, au point de l'envahir. Ses " trois pierres » sont, en fait, des constructions de terre tassée qui surélèvent le foyer lui-même et donnent à la maîtresse de maison la possibilité de cuisiner debout. Disposées en étoile, elles compartimentent la cuisine en trois espaces distincts ${ }^{7}$. 


\section{Pascale Dollfus}

Je pense à la générosité extraordinaire de Philippe pour ses étudiants, même tout à fait débutants, au temps et à l'énergie qu'il leur consacrait, à ses lectures si minutieuses de tous leurs écrits. Il avait le don de déceler dans les travaux de chacun des «idées ", qui souvent étaient encore très embryonnaires pour l'auteur lui-même. Exprimées par lui, elles devenaient claires, lumineuses, intelligentes. Jamais pourtant, il ne les aurait reprises à son compte.

Comme beaucoup d'étudiants qu'il a formés et épaulés, ses conseils, ses mots, m'accompagnent aujourd'hui encore quand j'écris.

4 Marie Lecomte-Tilouine

Philippe Sagant arrivait à son cours, joyeux, mains dans les poches, et se lançait avec passion dans l'évocation d'un monde qu'il savait rendre merveilleux : celui des Limbu du Népal oriental. Quel bonheur de se voir ainsi délivrer savoir et plaisir en lieu et place de l'habituel ennui qui présidait aux cours! Plaisir de la narration vive, mais aussi de l'ébullition d'une pensée, dont le charme particulier était fait d'un mélange de simplicité et de profondeur, d'un ancrage dans l'observation minutieuse du quotidien au service de grandes questions anthropologiques, telles que les fondements symboliques du pouvoir, l'expression spatiale de l'organisation sociale, la construction collective des trajectoires individuelles, les modalités de la colonisation des peuples indigènes et ses conséquences. Comme ses cours, les écrits de Philippe Sagant ne s'embarrassaient guère des tristes conventions académiques de l'époque et n'ont pas pris une ride. Ils ont cette rare qualité de transfigurer jusqu'aux sujets arides de l'anthropologie marxiste dont il s'est longtemps réclamé.

5 L'enthousiasme et la générosité de Philippe Sagant ont suscité chez ses étudiants une immense envie de progresser. Sa prise au sérieux de leurs premiers balbutiements et le flot d'idées qu'il leur délivrait en retour, le respect. Son humanité, l'excellence de sa matière ethnographique, portée par une écriture pétillante, ainsi que la liberté de sa pensée, ont fait de Philippe Sagant un vivant parmi les ombres du monde académique, hier, comme aujourd'hui.

\section{Satya Shrestha-Schipper}

J'ai rencontré Philippe Sagant pour la première fois à l'Université Paris X-Nanterre en 1992 alors que j'étais étudiante de maîtrise en ethnologie et qu'il donnait des cours sur l'Himalaya. Dès le début, nous nous sommes très bien entendus et, pour diriger mon mémoire, il a très gentiment accepté de m'encadrer. Son aide et ses encouragements ont été très importants pour moi. Il a passé énormément de temps à corriger le contenu de mon texte mais aussi sa forme et les fautes de français. Après cela, il a continué à diriger mes travaux de thèse jusqu' à ce qu'il tombe malade. J'ai longtemps refusé de changer de directeur, espérant son retour. 


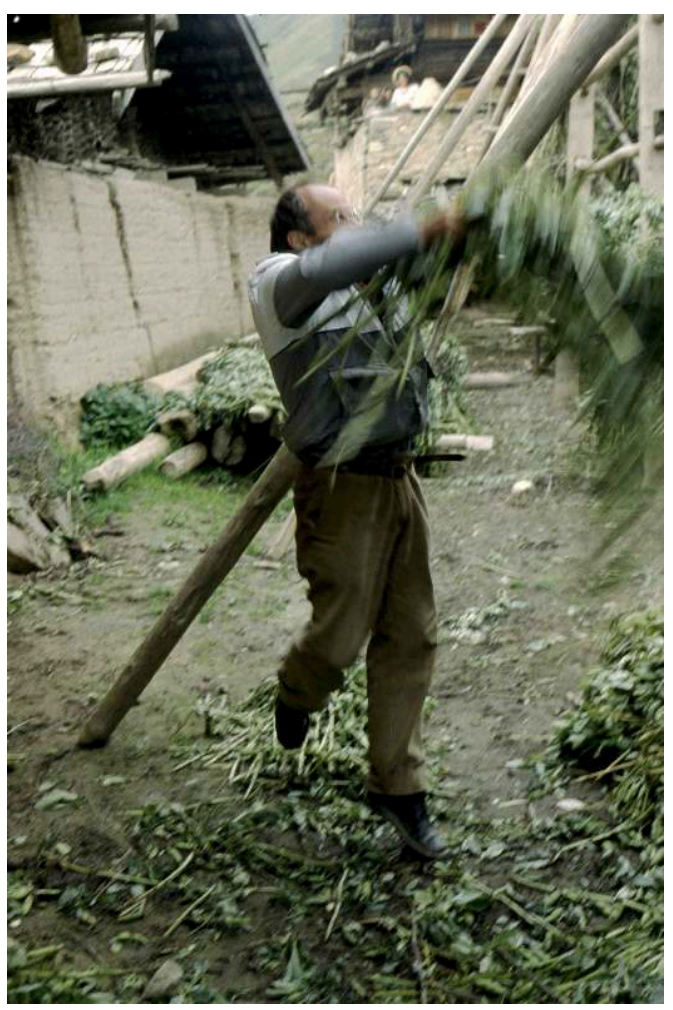

Katia Buffetrille (1986)

La fauche s'effectuait le mois du serpent, le neuvième selon le calendrier de Lhasa, c'est-à-dire en novembre. C'était un gros travail et pour en venir à bout, il fallait s'entraider. Un groupe passait d'abord dans le pré, il coupait l'herbe à la faucille et la déposait sur le sol derrière lui pour qu'elle sèche. Ensuite, il fallait confectionner de longues balles de fourrage en entortillant l'herbe coupée avec une sorte de tourniquet. On chargeait les balles à dos de yaks pour les descendre au village... ${ }^{8}$

\section{Grégoire Schlemmer, L'une des neuf forces de l'homme}

La grande influence intellectuelle qu'a exercé Philippe Sagant sur mon regard anthropologique est d'autant plus remarquable que je l'ai peu connu. Je n'ai suivi son séminaire à Nanterre sur le domaine himalayen que l'année qui précéda son terrible accident. Durant son séminaire, on quittait momentanément les structures abstraites de la pensée ou l'analyse formelle des systèmes de parenté pour aller, en compagnie des chamanes Yakthumba, rechercher les âmes égarées jusqu'au carrefour des quatre orients de l'autre monde, ou chevaucher avec les Sharwa et toute la grande caravane sur les haut plateaux tibétains, tout en redoutant une attaque des terribles Golok. Philippe Sagant était un conteur, et l'enthousiasme qu'il mettait dans ses propos se propageait à son auditoire. Et si ces histoires suffisaient à elles seules à éveiller l'attention, elles n'étaient pour autant qu'illustratives. Il ne s'agissait pas que d'anecdotes donnant chair à l'abstraite théorie. Elles révélaient aussi comment, dans ces sociétés qui ne sont pas tenues par un code législatif ou un corps de théoriciens, les discours se disent et les choses se font; comment paroles et actions se construisent dans leur réalisation. Plus qu'une technique narrative, plus aussi qu'un point de méthode, c'est toute une approche du terrain et une vision de l'anthropologie que révèle cette mise en récit dont Philippe Sagant avait le talent. Et ce ne fut là que l'une des nombreuses « forces de l'homme». 


\section{Stéphane Gros}

La passion communicative de Philippe Sagant pour l'ethnologie était la marque d'un enseignement à même de susciter des vocations. Philippe Sagant m'a ainsi initié à l'ethnologie et à des questionnements pour moi alors inédits. En tant que maitre, il avait cette aptitude rare et précieuse, par ses remarques sensibles, détaillées et stimulantes, de transmettre une analyse qui lui était propre, mais comme s'il n'avait fait que révéler une potentialité dans les travaux de ses étudiants. Il faisait ainsi participer à l'émergence d'une réflexion. Sa préoccupation pour le détail ethnographique et pour la mise en récit de la manière dont les idées sont vécues, selon ses mots, furent et continuent d'être un modèle et une source d'inspiration. Pour avoir contribué à la mise en ordre de ses archives, j'ai réalisé encore mieux l'ampleur de son travail et l'envergure de son ambition intellectuelle investie dans la poursuite d'idées maîtresses qu'il voyait plonger dans la nuit des temps, et qui soutenaient des édifices culturels pour beaucoup sur le point de disparaître.

\section{Gisèle Krauskopff}

Je retrouve une longue lettre de Philippe, simplement datée mercredi, dont je ne peux fixer la date : quatre longues pages d'une écriture ferme et élégante, sans la moindre rature; quatre pages détaillées sur la "malédiction » comme institution, sur les rituels des labours et des semis, leurs objectifs et la place qu'y tiennent les chefs de clans limbu. L'ethnographie est vivante, on y est, avec les témoins d'alors: «On dit que les semences tressaillent lorsque la force les pénètre. Les enfants se tiennent là les yeux écarquillés ». Mais si longtemps après, le plus extraordinaire pour moi, c'est que je ne peux me souvenir des questions, sans doute fort brèves, que j'avais pu poser, à l'origine d'un tel écrit, vraie matière d'un article. Une phrase pourtant l'ouvre : «ta lettre me ramène bien loin en arrière, dans le temps, dans l'exploitation des matériaux limbu. Elle me les fait voir d'un autre œil ». Elle dit cette générosité à l'égard des plus jeunes qu'il formait, cette écoute entière, au point de vous faire croire, vous débutant, à la valeur de vos manques. 


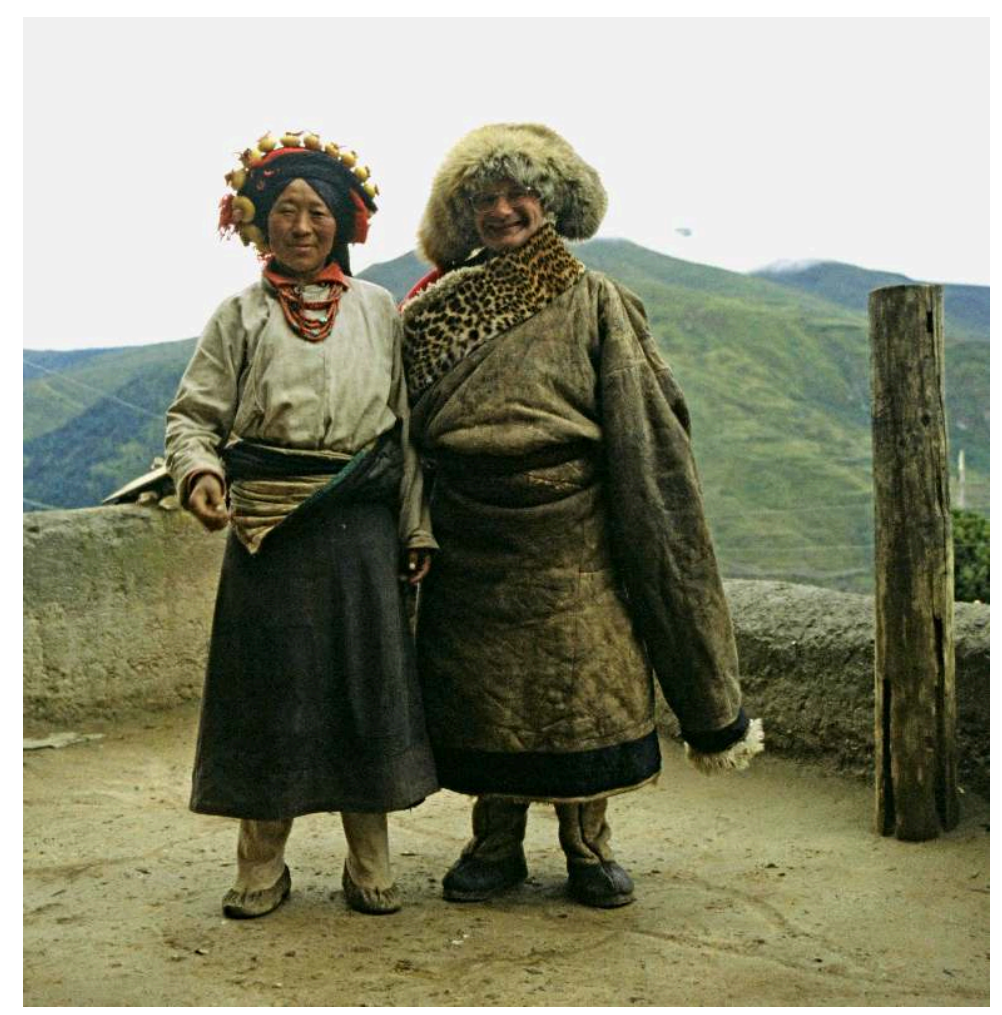

Katia Buffetrille (1986)

Chez les Sharwa, les hommes portaient des pelisses, chouba, faites de peaux de mouton, la toison à l'intérieur, au contact du corps, le col rehaussé de fourrure de léopard... Quand on voyait un homme avec une chouba de peaux d'agneau, on savait qu'il était riche, et qu'il partait l'hiver avec la grande caravane. ${ }^{9}$

\section{BIBLIOGRAPHIE}

\section{Bibliographie $^{10}$}

\section{Ouvrages et édition d'ouvrages}

1976 Le paysan Limbu, sa maison et ses champs (Paris, Mouton).

Karmay, S. G. \& Sagant, P.

1998 Les neuf forces de l'homme: Récits des confins du Tibet (Nanterre, Société d'Ethnologie).

Karmay, S. \& Sagant, P. (éds.)

1997 Les habitants du toit du monde : Études recueillies en hommage à Alexander W. Macdonald (Nanterre, Société d'Ethnologie).

1996 The dozing shaman : The Limbus of eastern Nepal. Scott, Nora B. (Trad.) (Delhi, Oxford University Press). 


\section{Articles}

1969 Les marchés en pays limbu (notes sur trois hat bajar des districts de Taplejung et de Terhathum), L'Ethnographie, pp. 90-118.

1969 Tampunma, divinité limbu de la forêt, Objets et Mondes, Print., 9, 1, pp. 107-124.

1970 Mariage par enlèvement chez les Limbu (Népal), Cahiers Internationaux de Sociologie, 48, pp. 71-98.

1971 L'exploitation de Séveyrac. Aperçu sur l'évolution économique récente d'une ancienne grange abbatiale, L'Aubrac : étude ethnologique, linguistique, agronomique et économique d'un établissement humain. Tome 2 : ethnologie historique (Paris : Éditions du C.N.R.S. Recherche coopérative sur programme organisée par le C.N.R.S), pp. 167-209.

1971 Note sur l'exploitation de Merlet, L'Aubrac : étude ethnologique, linguistique, agronomique et économique d'un établissement humain. Tome 2 : ethnologie historique (Paris : Ed. du C.N.R.S. Recherche coopérative sur programme organisée par le C.N.R.S), pp. 211-216.

1973 Les travaux et les jours dans un village du Népal oriental, Objets et Mondes, Hiver, 13, 4, pp. 247-272.

1973 Prêtres limbu et catégories domestiques, Kailash, 1, 1, pp. 51-75.

1975 Nationalité et État-nation : L'exemple de l'intégration politique des Limbu du Népal, Pluriel, 2, pp. 3-20.

Gallissot, R. \& Sagant, P., 1975 Les Limbu : groupe ethnique ou nationalité ?. Pluriel, 2, pp. 75-92. 1976 Les Limbu, population du Népal oriental, L'Ethnographie, 72, pp. 146-173.

1976 La Chine, vue d'un village himalayen, Critique, nov., 354, pp. 1186-1191.

1976 Becoming a Limbu priest : Ethnographic notes, in J. T. Hitchcock, R. L. Jones (éds), Spirit possession in the Nepal Himalayas (Warminster, Aris \& Phillips Ltd), pp. 56-99.

1978 Les pouvoirs des chefs limbu au Népal oriental, L'Homme, janv.-juin, 18, 1/2, pp. 69-107.

1978 Du village vers la ville et la plantation, L'Ethnographie, 77-78, pp. 11-33.

1978 Ampleur et profondeur historique des migrations népalaises, L'Ethnographie, 77-78, pp. 93-119.

1978 ‘Quand le Gurkha revient de guerre...', L'Ethnographie, 77-78, pp. 155-184.

1979 Le chamane assoupi, in M. Gaborieau \& A. Thorner (éds), Asie du Sud : Traditions et changements (Paris, Centre National de la Recherche Scientifique), pp. 243-247.

1980 Usuriers et chefs de clan : Ethnographie de la dette au Népal oriental, in La dette (Paris, Ecole des Hautes Études en Sciences Sociales), Purusartha, 4, pp. 227-277.

1981 Mais qui tuera le cochon dit ma mère ?, Objets et Mondes, 21, 2, pp. 83-86.

1981 La tête haute : maison, rituel et politique au Népal oriental, in G. Toffin, L. Barré, C. Jest (éds), L'Homme et la maison en Himalaya : Ecologie du Népal (Paris, Centre National de la Recherche Scientifique), pp. 149-180.

1981 Les gardiens de la banque : Histoires de vie et autres histoires, Tud Ha Bro, Sociétés bretonnes 6 (Rennes, Université de Haute Bretagne), pp. 21-39. 
1981 Le fils des Quatre Orients, Orients, Hommage à Georges Condominas (Paris, Sud-Est Asie-Privat), pp. 1-8.

1982 L'hindouisation des Limbu, in A. W. MacDonald (éd.), Les royaumes de l'Himalaya, histoire et civilisation (Paris, Imprimerie Nationale), pp. 165-239.

1982 Le chamane et la grêle, L'Ethnographie, 78, 87-88, pp. 163-174.

1982 L'espace limbu : Habitation et organisation du monde, Les Cahiers de l'ADRI 1, 6/7, pp. 13-28. 1987 La cure du chamane et l'interprétation des laïcs, in A. W. Macdonald (éd.), L'Ethnographie. Rituels Himalayens, 83, pp. 247-274.

1987 Traditions enfantines : L'apprentissage des techniques au Népal oriental, in B. Koechlin et coll. (éds), De la voûte céleste au terroir, du jardin au foyer (Paris : École Pratique des Hautes Etudes en Sciences Sociales), pp. 629-635.

1988 Le vol des connaissances, Autrement, Hors série 28, pp. 101-108.

1988 Le rapt d'une femme. Autrement, Hors série 28, pp. 113-121.

Karmay, S. \& Sagant, P. 1988 La place du rang dans la maison sharwa (Amdo ancien), in D. Blamont \& G. Toffin (Eds), Architecture, milieu et société en Himalaya (Paris, Éditions du CNRS, Études himalayennes 1), pp. 229-260.

1990 Les tambours de Nyi-shang (Népal) : Rituel et centralisation politique, in Tibet : Civilisation et société - Colloque organisé par la Fondation Singer-Polignac à Paris, les 27, 28, 29 avril 1987 (Paris, Fondation Singer-Polignac - Maison des Sciences de l'Homme), pp. 151-170.

Michailovsky, B. \& Sagant, P. 1992 Le Chamane et les fantômes de la malemort : Sur l'efficacité d'un rituel, Diogène, avr.-juin, 158, pp. 20-36.

1996 Les récits du chasseur, in C. Champion (éd.), Traditions orales dans le monde indien (Paris, EHESS), Purushartha 18, pp. 419-428.

1996 Dasai et le double pouvoir chez les Yakthumba, in G. Krauskopff \& M. Lecomte-Tilouine (éds), Célébrer le pouvoir : Dasai, une fête royale au Népal (Paris, Éditions du CNRS- Maison des Sciences de l'Homme), pp. 283-314.

Karmay, S. \& Sagant, P. 1997 Introduction, in S. Karmay \& P. Sagant (éds), Les habitants du toit du monde : Études recueillies en hommage à Alexander W. Macdonald (Nanterre, Société d'Ethnologie), pp. 9-30.

1999 Horse trading in Tibet, Nepal and India (1956-1959), traduit par Keyes Susan, European Bulletin of Himalayan Research, 15-16, pp. 30-31.

\section{Traductions en anglais}

1985 With head held high : The house, ritual and politics in east Nepal, Kailash,12, 3/4, pp. 161-222.

1988 The shaman's cure and the layman's interpretation, Kailash, 14, 1/2, pp. 5-40.

Michailovsky, B. \& Sagant, P. 1992 The Shaman and the ghosts of unnatural death : On the efficacity of a ritual, Diogenes, 158, pp. 19-37.

Comptes-rendus et à propos

1981 « Faith-Healers in the Himalayas » de C. J. Miller, L'Homme, janv.-mars, 21, 1, pp. 134-135. 
1982 « Le Corps Taoïste » de K. Schipper, Libération, 15 mars.

1983 « Migration and Ethnic Conflict in India » de M. Weiner, Annales, 2, pp. 299-300.

1984 « Ovibos, la grande aventure des hommes et des bœufs musqués » de R. Gessain,

L'Ethnographie, pp. 223-225.

1983 « Solstice Païen » de J. Y. Loude \& V. Lièvre, La Quinzaine Littéraire, 6 mai.

1985 « Les Indiens du Canada » de S. Hargous, L'Ethnographie, 1, pp. 155-157.

1985 « Voir, Savoir, Pouvoir, Le chamanisme chez les Yagua du Nord-Est péruvien », L'Ethnographie, 1, pp. 158-161.

1986 « Caste et classe en Asie du Sud (Purusartha 4) », J. Pouchepadass éd., Revue Française d'histoire d'outre-mer, 272, pp. 375-376.

1987 « Shamanism in Eurasia » de M. Hoppal (éd.), L’Homme, oct.-déc., 27, 104, pp. 123-125.

1988 « Le Mendiant de l'Amdo » de H. Stoddard, L'Homme, avr.-sept., 28, 106/107, pp. 357-359.

1992 « Lieu de neige et de genévriers : Organisation sociale et religieuse des communautés bouddhistes du Ladakh » de P. Dollfus, L’Homme, janv.-mars, 32, 121, pp. 206-208.

1996 « La chasse à l'âme. Esquisse d'une théorie du chamanisme sibérien » de R. Hamayon, L'Homme, avr.-juin, 36, 138, pp. 127-135.

\section{Traduction d'ouvrage}

En collaboration avec Gasiorowski, J., Traduction de Stomma, Ludwik, 1986 Campagnes insolites : paysannerie polonaise et mythes européens (Lagrasse, Éditions Verdier).

\section{Films}

En collaboration avec B. Genestier, 1973 En pays limbu, 16 mm., coul. 35 min., montage J. P. Lacam (Paris, Comité du film ethnographique).

En collaboration avec G. de Caune \& R. Cerre., S.d. Dans l'Himalaya, au rythme des saisons. Film En pays Limbu réduit à 13 min. pour la télévision, Antenne 2 .

En collaboration avec H. Heer, 1988 Reisende zwischen Himmel und Erde (Limbu). Hambourg, Multimedia.

\section{NOTES}

1. Alexander W. Macdonald, compte-rendu de P. Sagant «Le paysan limbu, sa maison et ses champs", L'Homme, 1979, 19(1), pp. 156-157.

2. Ramble Ch., 2008, The Navel of the Demoness (Oxford, Oxford University Press).

3. Sagant P., 1978, Les Pouvoirs des chefs limbu au Népal oriental, L'Homme, 18(1-2), pp. 106-107.

4. Karmay S. \& Sagant P., 1998, Les Neuf Forces de l'Homme. Récits des confins du Tibet (Nanterre, Société d'ethnologie), p. 74.

5. Le chamane assoupi, in M. Gaborieau \&@ A. Thorner (éds), 1979, Asie du Sud:Traditions et changements (Paris, Editions du CNRS), pp. 243-247.

6. Karmay S. \& Sagant P. 1998 Ibid., p. 38. 
7. Karmay S. \& Sagant P., 1987, La place du rang dans la maison sharwa (Amdo ancien), in D. Blamont \& G. Toffin (éds), Architecture, milieu et société en Himalaya (Paris, Éditions du CNRS), p. 238.

8. Karmay S. \& Sagant P. 1998, ibid., p. 118.

9. S. Karmay \& P. Sagant, 1998, ibid. 189.

10. Bibliographie compilée par Stéphane Gros et Grégoire Schlemmer à partir du fonds d'archive Philippe Sagant de la bibliothèque Eric de Dampierre (LESC, MAE - Paris 10 Nanterre). 